\title{
CapTUI: Geometric Drawing with Tangibles on a Capacitive Multi-touch Display
}

\author{
Rachel Blagojevic and Beryl Plimmer \\ Department of Computer Science \\ University of Auckland \\ Private Bag 92119 \\ Auckland, New Zealand \\ rachel.blagojevic@auckland.ac.nz, beryl@cs.auckland.ac.nz
}

\begin{abstract}
We present CapTUI, an innovative drawing tool that detects tangible drawing instruments on a capacitive multi-touch tablet. There are three core components to the system: the tangible hardware, the recognizer used to identify the tangibles and the drawing software that works in tandem with the tangibles to provide intelligent visual drawing guides. Our recognizable tangible drawing instruments are a ruler, protractor and set square. Users employ these familiar physical instruments to construct digital ink drawings on a tablet in an intuitive and engaging manner. The visual drawing guides enhance the experience by offering the user helpful cues and functionalities to assist them to draw more accurately. A user evaluation comparing CapTUI to an application with passive tools showed that users significantly preferred CapTUI and found that the visual guides provide greater accuracy when drawing.
\end{abstract}

Keywords: TUI, tangible, multi-touch, physical interaction, capacitive, drawing tools.

\section{Introduction}

CapTUI is an innovative drawing application for capacitive multi-touch tablets that detects tangible drawing instruments. To develop CapTUI we have designed and constructed tangibles, developed a recognizer to detect the tangibles on a capacitive touch display, and built drawing software that works in tandem with the tangibles to provide intelligent visual drawing guides.

A skill acquired very early in life is the ability to manipulate tangible objects. As a case-in-point, mathematics education has long used manipulatives for introducing young children to mathematical concepts as their intuitive understanding of the physical enables them to transition into the realm of the conceptual [1-3]. Using tangibles for computing operations takes advantage of this existing skillset: pen-based interaction is a testament to this. Tangibles lower the level of interaction abstraction allowing users to apply their natural tool-based skillset to the digital environment. The use of tangibles on touch displays has been shown to improve interaction with interface objects as they are easier to manipulate, acquire and control in comparison to virtual objects [4]. 
Though the stylus has long been available as a drawing instrument for digital canvases, other instruments have not yet been explored. In addition to stylus input, designers often use passive tools such as rulers or French curves on tablets to guide drawing. Converting these passive tools to active tools seems a promising evolution for drawing based content creation. We have created three recognizable tangible drawing instruments: a ruler, protractor and set square. Through our application, users are able to apply these familiar physical instruments to construct digital ink drawings on multi-touch tablets. Transforming passive instruments into active tools, as we have done here, allows further intelligence to be built into the system. To our knowledge, recognizable tangible drawing tools (other than styli) have not been used on capacitive touch displays for creating electronic content.

Touch surfaces are a well-established technology. Small touch sensitive devices, e.g. phones and tablets, typically have capacitive displays, larger touch devices, such as tabletops, have camera-based detectors. Tangibles on tabletops have been explored for many years e.g. [5-8]. These systems typically use cameras to capture the position of the tangible; this requires specialist equipment and environments. The advantage of capacitive touch is that it is built into many readily available tablets that are portable and require no special environment. Using capacitive touch alters the recognition approach from image processing to gesture processing of the touch points. Touch points may be fingertips or conductive material [9] given that capacitive displays detect touch via the electrical properties of fingertips. As an extension of recent techniques [9] we have devised a novel approach which enables tangible drawing tools to work with capacitive displays (Fig. 1).

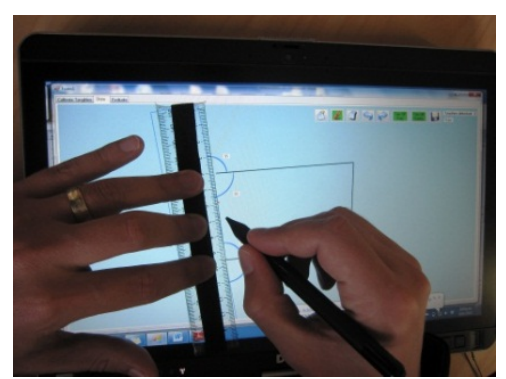

Fig. 1. CapTUI

To our knowledge tangible geometry drawing tools have not been used in this context before. There are two key contributions of this research: the design and construction of tangible drawing tools for capacitive touch surfaces, and visual drawing guides to enhance the geometric drawing experience. Together, these enhancements have the potential to provide benefits over using passive drawing tools on a screen. In a user evaluation, we explore this idea by comparing our prototype, CapTUI, to drawing with passive tangibles. Possible areas of application for our system include design fields such as architecture and mathematics education.

\section{Related Work}

Physical objects are beneficial for learning [10,11]. For example, manipulatives have been used for years to assist in mathematics education [1-3]. Similarly tangibles are a particularly useful tool for children when interacting with technology because of the added physicality $[4,12,13]$. Other advantages of tangibles include increasing explorative behaviour [14], reducing conflicts in cooperation [15, 16], encouraging 
prolonged engagement $[17,18]$ and acting as a useful aid to problem solving in comparison to standard graphical user interfaces [19]. Tangibles have been used to enhance physics and mathematics learning [20, 21] while other e-learning tools link geometry with algebra in order to augment the learning experience [22, 23].

The use of tangibles on touch surfaces has been shown to improve interaction with interface objects. Tuddenham et al [4] compared the use of tangibles and virtual objects on multi-touch interfaces. They found that when using the tangible objects significantly fewer errors were made and significantly less time was taken to acquire and manipulate the objects.

Limited work has been done in the area of tangibles on capacitive touch screens. SmartSkin is an early system that used capacitive sensing with a mesh shaped antenna to detect hands and objects [24]. Conductive materials were attached to blocks in patterns to allow objects to be identified. Yu et al [9] present three tangible technologies for use on capacitive touch surfaces: spatial, frequency and hybrid. Their spatial tangibles employ patterns for object identification, from at least four touch points. Four touch points can be limiting if users have multiple tangibles on touch surfaces that are limited in the number of touch points they are able to detect. Frequency tangibles use a modulation circuit to generate touches of varying frequency. They only require one touch point; however they rely on a power source, are unable to detect object orientation, and are limited in tracking fast movements. The hybrid tangible combines the spatial and frequency technologies to address the aforementioned issues. Our observation is that the tangibles they have presented are quite large in size - particularly thickness, in comparison to our tangibles. They report that the size of the circuit board alone is $2 \times 3 \times 3 \mathrm{~cm}$. AppMATes ${ }^{1}$ are another example of tangible objects, in the form of toy cars that can be used on the Apple iPad. The cars are uniquely identifiable using touch point patterns. More recently [25] stackable tangibles, sliders and dials for capacitive screens have been introduced. The stackable tangibles are able to sense changes in capacitance when blocks are placed on top of them - this in turn modifies the touch point pattern for identification. The dials and sliders are made of conductive zebra rubber and also use unique touch point patterns for identification.

Very few studies have been performed testing the usability of tangibles on capacitive displays. One study compared CapWidgets [26], tangible dials designed for mobile capacitive screens, to touch dials. The results showed that touch was significantly faster and rated higher for usefulness, satisfaction, ease of use, and learnability but there was no significant difference in precision. They identified aspects of the design of the tangibles and the software to be the main hindrance to the usability of their system. Jansen et al [27] designed tangible remote controllers for wall sized displays. The tangibles are manipulated on a capacitive tablet as a way of interacting with a wall sized display. The tangibles were stuck to the tablet to allow mobility and to reduce the need for visual attention. They used various conductive materials including conductive foam and ink to construct the tangible sliders and dials. Their user study compared tangible and touch interaction for a slider. Overall they found that using the tangible slider produced fewer errors, was faster to acquire and did not

\footnotetext{
${ }^{1}$ http: / / www . appmatestoys.com/
} 
require as much visual attention as the virtual slider. However, they did comment that their tangible design "still does not measure up to commercial physical controls".

Geometric drawing applications are common. Most rely on mouse and keyboard input [22, 23]. Sketchpad [28] presents the earliest work using a light pen to construct line drawings. It includes the ability to apply geometric constraints to drawings. Balakrishnan et al [29] introduced the concept of drawing on a large scale with digital tape. Tangible rulers for camera-based systems have been used [4, 30] but their focus has been on investigation of object acquisition or positioning rather than how the tangible might facilitate drawing and precise measurement.

Although the use of tangibles and multi-touch has been investigated in the context of camera-based surface interaction, tangible drawing tools with capacitive screens is yet to be explored. The interaction with drawing tools differs from the dials, blocks and sliders from previous work in tangible user interfaces (TUI's) for capacitive screens. Of the few studies that have been performed it is evident that the tangible's design is the key challenge to success [26, 27]. The CapWidgets study [26] suggests that tangible design was a hindrance to the usability of their system, while [27] also allude to the difficulty of constructing good tangibles. The combination of tangible drawing tools with capacitive screens presents significant challenges in the hardware and the interaction design of the applications to ensure a good user experience.

\section{Our Approach}

CapTUI combines recognizable tangible drawing tools with an intelligent drawing application. In this section the tangible hardware design is described, followed by the recognition approach used for these tools. Section 3.3 provides details on the drawing application and finally Section 3.4 describes CapTUI's visual drawing guides.

\subsection{Tangible Hardware}

Our goal is to create easily manipulated drawing instruments that are recognizable on a capacitive touch display. Capacitive screens are designed specifically to detect the electrical properties of fingertips. Hence we use conductive materials that act as an extension of the fingertips for the touch point connections between the tangibles and the touch display. These touch points are electrically connected to the user's body via his or her fingers as the user's body is the source of the electrical current.

To discriminate between the tangibles each requires a unique ID. The touch points on the bottom of each tangible are placed so as to form unique patterns. This ID allows differentiation between tangibles, and calculation of a tangible's orientation and location (see Section 3.2 for a full explanation).

There were several iterations in our design process for constructing the tangible hardware. Off-the-shelf standard drawing tools were modified by adding conductive materials to allow detection on the capacitive touch screen. Fig. 2 shows some of our designs using various conductive materials such as: aluminum foil/tape, conductive rubber, conductive foam, steel nuts and bolts, copper wire, and conductive ink [31]. 

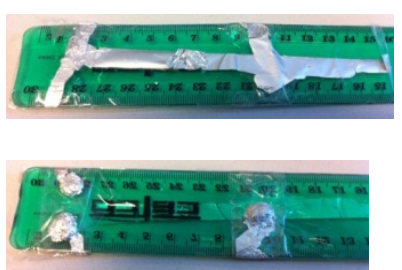

a) Aluminium foil and tape.

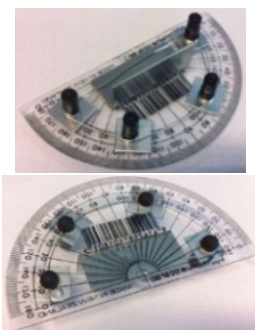

d) Metal washers, copper wire, conductive rubber and metal handle.

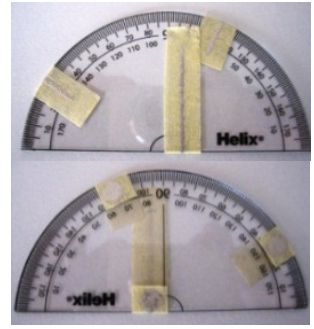

b) Conductive ink.

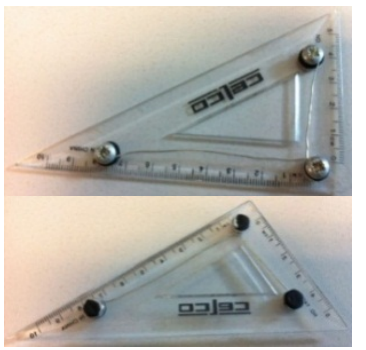

c) Steel nuts and bolts, copper wire and conductive rubber.

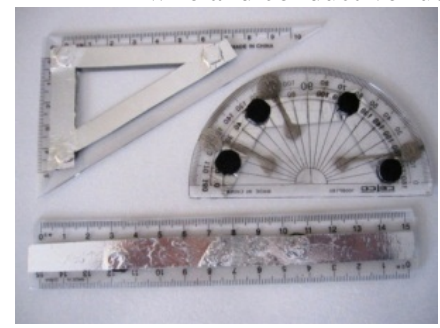

e) Conductive foam and aluminum tape.

Fig. 2. Tangible hardware designs

After preliminary testing of these prototypes, the following factors affecting the detection and use of the objects were identified.

- As a whole, the materials used must ensure a consistent circuit can be maintained, i.e. all circuit components are well connected so that the current from the user's fingertips has an unbroken path to the screen. We found when several materials are used, and therefore more connections required, a significant amount of pressure had to be applied before the touch points were detected.

- Tangible stability is important to maintain consistent contact between the screen and the tangible. This means that the touch points must be as flat as possible and positioned such that when the tangible is moved across the screen all points stay in contact with the surface. If the tangible is unstable, and a touch point loses contact with the screen then the tangible is no longer detected.

- To maintain tangible to body contact, there must be a clear point of contact for the user's fingertips when the tangible is in use. Our final prototype's top surface is covered with conductive material (Fig. 3).

- Usability of the tangible is poor if there is too little friction between the screen and the tangible. Using materials that add friction between the tangible and the screen, reduces unwanted movements when drawing, allowing users to draw along the tangible easily without having to concentrate on keeping it steady.

- Designs that require the conductive material to pass over the edge of the tangible block the user from drawing comfortably on the tangible edge (such as the prototypes shown in Fig. 2(a) and (b)). 
Table 1. Tangible hardware designs vs design factors

\begin{tabular}{lccccc}
\hline & $\begin{array}{l}\text { Consistent } \\
\text { circuit }\end{array}$ & Stable & $\begin{array}{l}\text { Clear point } \\
\text { of body } \\
\text { contact }\end{array}$ & $\begin{array}{l}\text { Friction } \\
\text { with } \\
\text { screen }\end{array}$ & $\begin{array}{l}\text { Comfortable draw- } \\
\text { ing (material does } \\
\text { not pass over edge) }\end{array}$ \\
\hline $\begin{array}{l}\text { a) Aluminium foil and tape } \\
\text { b) Conductive ink }\end{array}$ & $\checkmark$ & $\checkmark$ & & $\checkmark$ & \\
\hline $\begin{array}{l}\text { c) Steel nuts and bolts, } \\
\text { copper wire and conduc- } \\
\text { tive rubber }\end{array}$ & & & & $\checkmark$ & $\checkmark$ \\
\hline $\begin{array}{l}\text { d) Metal washers, copper } \\
\text { wire, conductive rubber } \\
\text { and metal handle }\end{array}$ & & & $\checkmark$ & $\checkmark$ & $\checkmark$ \\
\hline $\begin{array}{l}\text { e) Conductive foam and } \\
\text { aluminium tape }\end{array}$ & & $\checkmark$ & $\checkmark$ & $\checkmark$ & $\checkmark$ \\
\hline $\begin{array}{l}\text { Final design } \\
\text { (conductive foam) }\end{array}$ & $\checkmark$ & $\checkmark$ & $\checkmark$ & $\checkmark$ & $\checkmark$ \\
\hline
\end{tabular}

Table 1 shows the performance of each tangible design in terms of the design factors listed above; where performance is based on expert judgement and informal testing. Design (a) was a proof of concept to show that recognition was possible; however it failed to meet most of the design criteria. Design (b) provided a consistent circuit and was a stable design, however its main drawback was that the conductive ink had to be drawn on tape to ensure the circuit was maintained over the edge of the tangible. An experiment using the ink without tape failed as the edge of the tangible was so thin it was difficult to maintain an unbroken circuit. Design (c) and (d) involved too many materials and therefore made it difficult to maintain a consistent circuit. The conductive rubber touch points, while providing a good amount of friction with the screen, were difficult to mould and therefore did not provide a stable base for the tangibles. Design (e) had conductive foam for the touch points which improved stability, as they are easier to cut into shape, and maintained friction with the screen. However it was difficult to maintain good circuits as there were still two conductive materials in use.

Our final design (Fig. 3) was constructed based on the identified design factors; these included a ruler, set square, and protractor. One conductive material was used, conductive foam. This reduces the number of connections that must be made to maintain a circuit from the user's fingers to the screen. The foam covers a large portion of the top surface of the tangible so that there is a clear point of contact for the user's fingers. The foam provides a good amount of friction so that the tangible does not make unwanted movements on the screen. It also provides a stable base for the tangible so that the touch points are consistently in contact with the screen. In addition the conductive foam is easier to work with than other materials as it can be cut into the shape required and does not have to pass over the edge of the tangible. 

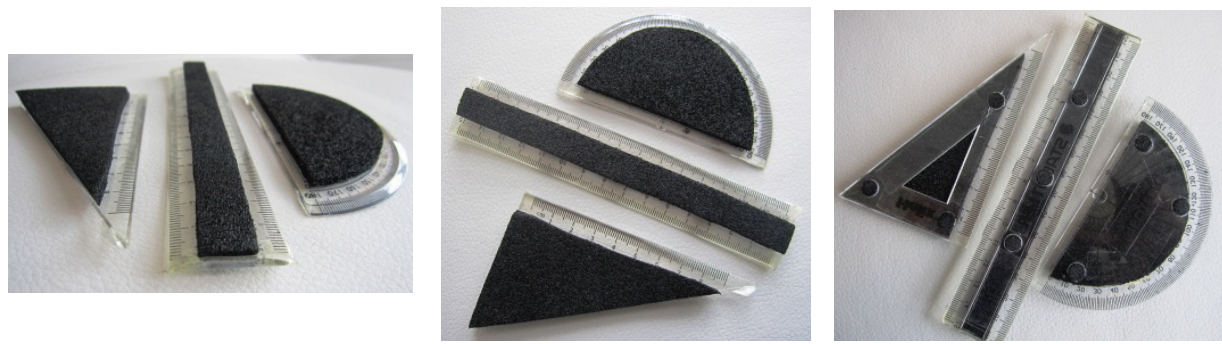

Fig. 3. Final tangible drawing tools (conductive foam)

\subsection{Tangible Recognition}

This section describes how each tangible can be identified and its position computed. The recognition approach is similar to that of SmartSkin [24]. Recognition of the tangible allows for further intelligence to be added to the tangible interaction as described in Section 3.4.

The tangible ID patterns are based on the distance between each pair of points. When constructing a pattern the distances must be distinct; for example, a pattern that forms an isosceles triangle is not allowed because it has two or more identical distances between points. Identical distances make it impossible to determine the correct orientation of the tangible, with the obvious exception of regular shapes (e.g. circle).

There are two main phases for the recognizer: learning and recognition. The learning phase allows users to register and calibrate the tangibles so that they are identified by the system. Several details about each tangible are stored: the location of the touch points, the distances between touch points (i.e. its unique ID), the outline points of the tangible and the type of tangible (ruler, protractor or set square) as specified by the user. This data is used later to identify the tangible and display its outline on the screen.

Tangibles only need to be registered with the system once. To register the tangible (Fig. 4), the user places it on the registration screen and moves the guidelines to delineate a bounding box. The user then specifies the type of tangible (ruler, set square, protractor) from a list.

During the recognition phase, touch input from the tangible currently in use is passed to the recognizer. The recognizer finds its ID by calculating the distances between each touch point detected and comparing these to the previously saved distances for each calibrated tangible. The recognition adds (or subtracts) a degree of error tolerance $(e)$ when comparing the distances between the detected touch points and the saved set of distances. The error tolerance is required as capacitive hardware detects an area of touch but only reports the centre point position. This means the precise position of a touch point can be difficult to determine as we have no way of knowing which part of the tangible's touch point has been detected as the centre. We experimented with different sized touch points and found that a diameter of approximately $7 \mathrm{~mm}$ is the smallest size that can still be detected on the touch screen for our tangibles. 


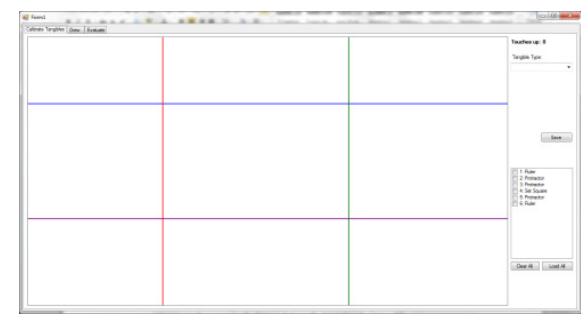

Fig. 4. CapTUI tangible calibration interface

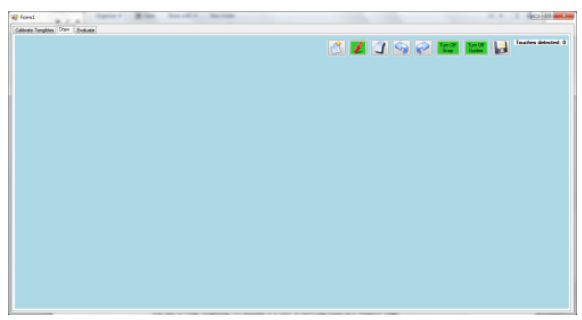

Fig. 5. CapTUI drawing interface

The tangible identification algorithm is as follows:

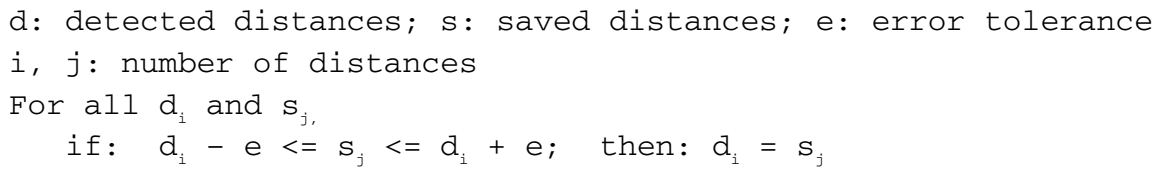

If a match is found between all $d_{i}$ and $s_{j}$ then the tangible ID is identified as s. Our informal experiments showed that a $2 \mathrm{~mm}$ tolerance level is sufficient for maintaining a good rate of recognition.

The advantage of including a level of tolerance for the patterns is that $100 \%$ accuracy can be achieved by the recognizer, resulting in better usability. There is a tradeoff here, as calculating the position and orientation of the tangible is less accurate. To minimize the effect of this tradeoff the user is provided with a visualization of the tangible on the screen; this is described further in Section 3.4.

\subsection{Drawing Application}

The tangible hardware and recognition algorithm were combined together in a drawing application (Fig. 5). Our application allows the tangible ruler, protractor and set square to be used to construct drawings on a capacitive multi-touch screen. It also includes basic functions such as save, erase, undo and redo.

To construct drawings using a tangible drawing tool the user slides a stylus along the edge of the tangible that is placed on the screen (Fig. 1). As the user draws, beautified lines (or curves) are rendered.

The application was developed using the .NET 4.0 framework on a Windows 7 Dell Latitude XT3 tablet, Intel core i7-2640M with 8GB RAM, built in stylus and four available touch points.

\section{Ink Beautification}

One of the main purposes of having geometric tools in drawing is to use the tangibles as a guide for precise drawing. However, due to the imprecise nature of contact points on the screen, the user's ink may not be rendered where they intend. Therefore we beautify the ink to try to produce what the user intends, in a similar way to many digital ink drawing programs such as [32]. Our system includes two forms of ink beautification: ink to tangible edge snapping and ink corner snapping. 


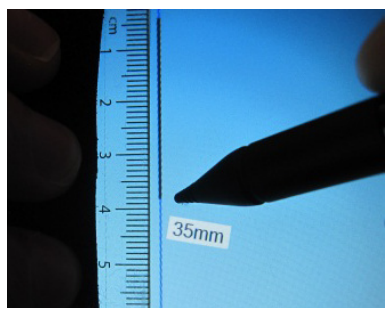

Fig. 6. Ink-to-edge snapping and length visualization

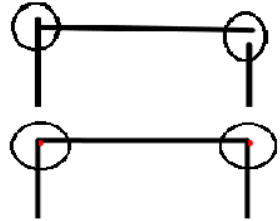

Fig. 7. Corner snapping

For ink-to-edge snapping, we assume if a user is drawing close to a tangible edge, they intend to draw right on the edge. Therefore ink points are dynamically translated so that they snap to the edge of a tangible's virtualized outline as they are drawn in real time. The result is a beautified straight/curved line drawn along the edge of the tangible (Fig. 6).

Corner snapping beautifies ink to render clean intersections between strokes that are close by. It is automatically enabled in two situations. The first connects the starting point of a stroke to the end of existing strokes when the user first starts drawing a stroke. The second connects the stroke end point to other strokes (at any position) once the stroke is completed. The beautification is applied to the newly added stroke and existing strokes; both strokes are extended or reduced such that they meet exactly at an intersection point (Fig. 7). A corner is snapped if the current stroke end points are close to another stroke - this requires a distance threshold to be set for determining 'closeness'. As this is heuristic-based, corner snapping can be reversed using the undo function. It can also be turned off if the user desires.

\subsection{Visual Drawing Guides}

Our early experiences suggested that drawing guides are essential for an enjoyable experience on current touch technologies [33]. The visual drawing guides we developed assist the user in constructing accurate geometric drawings. These guides are directly dependent on the unique identification of the tangibles; therefore making them only possible and relevant with recognizable tangibles.

\section{Tangible Outline}

When the tangibles are detected on the screen a visualization of the tangible outline is displayed (the blue line in Fig. 8). The visualization is rendered at the correct location and orientation, using the tangible recognition information, so as to match the tangible itself, though with a small offset such that the outline is visible.

\section{Length Visualizations}

When drawing straight lines using the ruler or set square, an adjustable line length function is enabled. As a stroke is drawn along the edge of a tangible, its length can be adjusted by moving the stylus up and down the edge. This function has two main 
advantages: when the user draws the line further than the intended length it can be easily corrected, and having a line dynamically adjustable in length provides a prototyping experience where the user can see how the line will look at various lengths.

To assist in solving occlusion issues caused by the tangible, stylus or hand on the display, the length visualization displays line length in millimetres as the user draws with a ruler or set square (Fig. 6). This visualization is supplementary to the markings on the tangible. The visualization works in tandem with the adjustable length function by providing real time stroke length feedback.

Without recognition of the tangible these visualizations would not be suitable functions as there would be no way of knowing that the user intends to draw a straight line as they are completing the action. When the ruler or set square is recognised we can be sure that straight lines are desired.

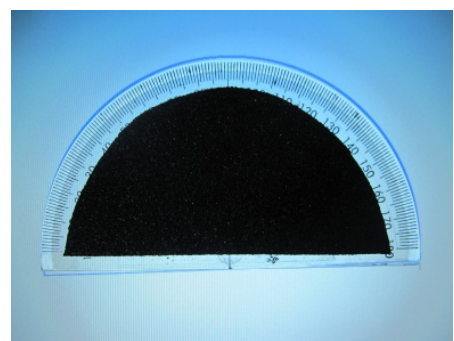

Fig. 8. Tangible outline

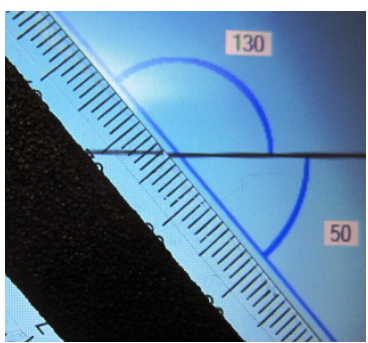

Fig. 9. Angle visualizations

\section{Angle Visualization}

The angle visualization displays the angle between existing strokes and the edges of a tangible ruler to allow a line to be drawn intersecting other lines at specific angles (Fig. 9). It is created by detecting intersections between existing strokes and the ruler edges, calculating the angle between these lines, and displaying the results. This enables the construction of various geometric shapes which consist of connected lines of specific angles, for example, regular polygonal shapes. We have also included an affordance which freezes the angle in place if it reaches multiples of five or ten degrees to assist the user in obtaining common angles. If the angle has been frozen, it is only unfrozen if the tangible is moved approximately 5 degrees away. This threshold was chosen after some informal testing. In essence, this visualization can replicate the protractor for measuring angles. However, it relies on the recognition of the ruler so that the angles between the tangible ruler and existing digital ink can be calculated in real time.

In a similar way to the length visualization, an angle visualization displays the angle in degrees as the user draws an arc with the protractor. This helps to solve occlusion issues and supplements the markings on the tangible itself by providing real time feedback. Again, this visualization relies on the recognition of the protractor to confirm that the user intends to measure angles. 


\section{$4 \quad$ Evaluation}

The objective of this evaluation is to determine if CapTUI assists users to easily draw precise geometric drawings. In particular, we are interested in comparing drawing with CapTUI's recognizable tangibles to drawing with standard non-recognizable drawing tools on a screen. For this comparison we have developed another drawing application, referred to as Paint. Paint can be used to construct geometric drawings in much the same manner as CapTUI except that it does not recognize the drawing tools on the screen. To make the comparison as fair as possible it includes the same beautification and corner snapping functions as CapTUI. However, it does not include CapTUI's tangible outline, angle and length visualizations, as these functions are dependent on tangible recognition. Using this comparison we can determine if recognizable tangibles and visual guides, that are possible as a result of this recognition, assists users when constructing precise geometric drawings.

A within-subject design was used where each participant was given the same tasks to complete using CapTUI and Paint; half used CapTUI first and the other half used Paint first. Participants first completed a pre-questionnaire on their previous experience with touch, stylus and drawing applications. They were then given an introduction to the first system and time to familiarize themselves with the application. When ready, participants completed the tasks required using the first system and filled in a questionnaire on their experience. This process was repeated for the second system.

There were seven tasks designed for the evaluation; the first three tasks served as training tasks (Fig. 10). Each training task aimed at familiarizing the participant with a different drawing function or tangible. They were not told that they were training tasks: this was done to try to encourage them to complete the tasks with the same amount of effort they might apply for the real tasks and therefore get the most out of the training. The remaining four tasks comprised the evaluation tasks, providing data for our analysis (Fig. 11). The focus of task one was on connecting lines of specific length. Task two was aimed at evaluating drawing lines at a specific angle and use of the protractor to connect an arc. Task three focused on drawing angles of specific magnitude. In task four, angle and length measurements were required concurrently.

Quantitative metrics used to analyse the data collected from these tasks included the time taken, and average length and angle errors. Errors were counted as $1 \mathrm{~mm}$ or 1 degree away from the required measurement. The average length and angle error was calculated for each task using the sum of the errors made / number of lengths or angles in the task. Participants also completed a questionnaire to record their experiences using each system. Questions were presented using a 5 point Likert scale, except for a small number of open ended questions. A Wilcoxon Signed Ranks Test was used to test for significant differences in the results, unless otherwise stated.

After a pilot study with two participants, twelve participants (eight males and four females) were recruited for the final study. They came from a range of backgrounds including computer science, law and health. Seven participants used touch interfaces frequently; the remainder had used such interfaces a few times or occasionally. The majority of participants had experience using stylus input before. Six participants had used a drawing program on a touch device before. 

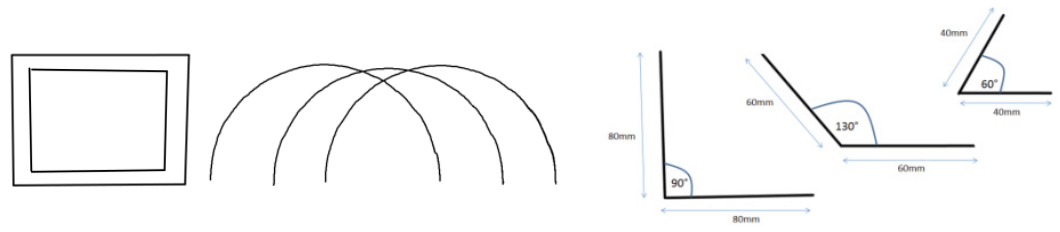

Fig. 10. Training tasks

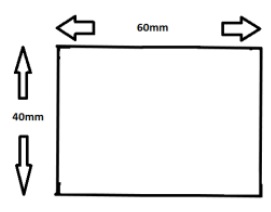

Task 1

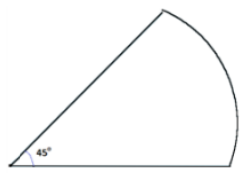

Task 2

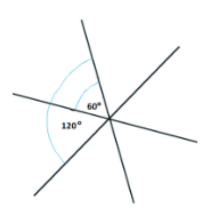

Task 3

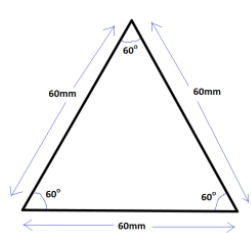

Task 4

Fig. 11. Evaluation tasks

\subsection{Results}

Participants were asked to give an overall rating for each system (using a 5 point Lik ert scale). Seven participants rated CapTUI higher than Paint, one rated Paint higher and four considered them equal. All participants rated CapTUI as good, very good or excellent $(\mathrm{m}=3.75$, s.d. $=0.62)$ (see Q1, Table 2). Ratings for Paint were more varied $(\mathrm{m}=2.83$, s.d. $=1.11)$. The overall ratings were found to be significantly different $(\mathrm{z}$ $=-2.157, \mathrm{p}=0.031$ ) where participants rated CapTUI significantly higher than Paint.

The results for the quantitative measures are as follows. A paired t-test showed that there was no statistically significant difference $(\mathrm{t}=1.644, \mathrm{p}=0.107)$ in the time taken to complete the tasks between using Paint $(\mathrm{m}=58.30$, s.d. $=32.78)$ and CapTUI $(\mathrm{m}=$ 70.36, s.d. $=32.63$ ), Fig. 12. This result is favourable for CapTUI as it shows that even with the time taken for detecting the drawing tools it did not take significantly longer to complete the tasks. There was no significant difference $(\mathrm{z}=-1.029, \mathrm{p}=$ $0.304)$ in average length errors per task between using Paint $(\mathrm{m}=1.79$, s.d. $=2.67)$

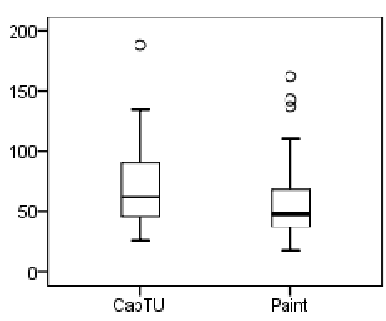

Fig. 12. Time taken to complete tasks (seconds)

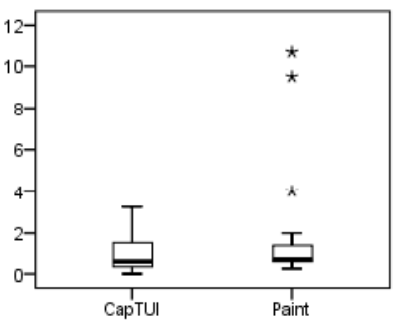

Fig. 13. Average length errors

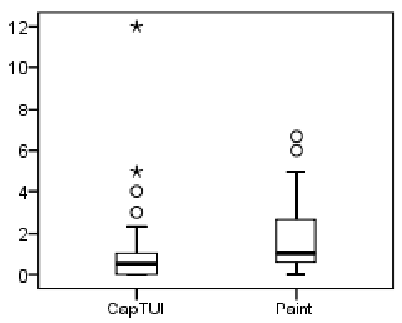

Fig. 14. Average angle errors 
Table 2. Comparative Questionnaire Results (1st row: CapTUI, 2nd row Paint)

\begin{tabular}{lccccc}
\hline & Poor & Fair & Good & Very Good & Excellent \\
\hline Q1. Overall rating & 1 & 4 & 4 & 2 & 1 \\
\hline \multicolumn{1}{l}{ Q2. Drawing tools ease } & & 1 & 5 & 5 & 1 \\
$\begin{array}{l}\text { of use } \\
\text { Q3. Drawing tools }\end{array}$ & 1 & 1 & 2 & 7 & 1 \\
\hline usefulness & 1 & & & 8 & 4 \\
\hline $\begin{array}{l}\text { Q4. Ease of length } \\
\text { measurement }\end{array}$ & 1 & 2 & 2 & 5 & 2 \\
\hline Q5. Ease of angle meas- & & 1 & 1 & 7 & 3 \\
urement & 2 & 3 & 1 & 4 & 2 \\
\hline Q6. Drawing accuracy & & 1 & 4 & 5 & 2 \\
& 1 & 5 & 1 & 4 & 1 \\
\hline Q7. Drawing tidiness & & & 4 & 5 & 3 \\
\hline Q8. Enjoyment & 1 & 6 & 1 & 3 & 1 \\
\hline
\end{tabular}

and CapTUI $(\mathrm{m}=0.95$, s.d. $=0.82)$, Fig. 13. This shows that both Paint and CapTUI were comparable when creating lines of accurate lengths. On the other hand, average angle errors per task were significantly less $(\mathrm{z}=-2.861, \mathrm{p}=0.004)$ when using CapTUI $(m=0.96$, s.d. $=1.91)$ than when using Paint $(m=1.68$, s.d.=1.63), Fig. 14. This result confirms that angles drawn using CapTUI were significantly more accurate.

The following are the results obtained from questions answered using a 5 point Likert scale (see Table 2). Participants enjoyed (Q8) using CapTUI $(\mathrm{m}=4.33$, s.d.= $0.49)$ significantly more than using Paint $(\mathrm{m}=3.25$, s.d.=1.06), $(\mathrm{z}=-2.288, \mathrm{p}=$ 0.010). All participants agreed that the drawing tools were useful (Q3) when completing the tasks for CapTUI $(\mathrm{m}=4.33$, s.d. $=0.49)$; however for Paint the results were more varied $(\mathrm{m}=3.92$, s.d. $=1.16)$. There was no significant difference found between these results $(\mathrm{z}=-0.877, \mathrm{p}=0.380)$. There was no significant difference found for the responses when asked if the drawing tools for each system were easy to use $(\mathrm{Q} 2, \mathrm{z}=-0.272, \mathrm{p}=0.785)$. This indicates that the recognizable drawing tools were just as easy to use as the non-recognizable tools. When asked which system is easier to use overall, five participants chose CapTUI, three chose Paint, and four considered them equal. The main reason for choosing CapTUI was that the visual guides made the system much easier to use than Paint ("the angle and length indicators were really 
helpful", "easier especially for the more technical drawing due to the ability to easily get exact lengths and fairly easily get precise angles"). The main reason for not choosing CapTUI was due to inconsistent tangible detection ("CapTUI was more accurate and tidy but I had a bit of problem with adjusting the tools").

All but one participant agreed that it was easy to measure line length (Q4) with CapTUI $(\mathrm{m}=4.42$, s.d. $=0.90)$ in comparison to seven participants for Paint $(\mathrm{m}=$ 3.41 , s.d. $=1.24)$, however this difference was not found to be significant $(\mathrm{z}=-1.796$, $\mathrm{p}=0.072)$. Ten participants agreed that it was easy to measure angles (Q5) with CapTUI $(\mathrm{m}=4.00$, s.d. $=0.85)$, compared to only six participants for Paint $(\mathrm{m}=3.08$, s.d. $=1.44)$; this was also not statistically significant $(\mathrm{z}=-1.530, \mathrm{p}=0.126)$. It is possible that with a larger sample size statistically significant differences may be found for these factors given that the majority of participants preferred CapTUI for ease of line and angle measurement.

Eight participants agreed that their drawings were tidy (Q7) when drawn using CapTUI $(m=3.92$, s.d. $=0.79)$, compared with four participants for Paint $(m=2.75$, s.d. $=1.22)$. These differences were statistically significant $(\mathrm{z}=-2.124, \mathrm{p}=0.034)$. Only one participant did not believe their drawings were accurate (Q6) with CapTUI $(\mathrm{m}=3.67$, s.d. $=0.89)$, compared with half of participants with Paint $(\mathrm{m}=2.92$, s.d. $=$ $1.24)$, however this difference was not statistically significant $(\mathrm{z}=-1.562, \mathrm{p}=0.118)$. When asked about the overall accuracy of each system, eleven participants considered CapTUI to be the more accurate of the two systems; this was attributed to the visual guide functionalities, in particular the angle indicators ("aids to angles were helpful in making accurate shapes", "accurate and faster to use given the angle/length indicators", "the onscreen angle calibration and tool recognition made it much easier to draw accurate shapes").

Several questions were asked specifically about CapTUI (using a 5 point Likert scale). All but one participant agreed that learning to use the tangibles was easy. Five participants disagreed that the recognition of the tangibles was good. This aspect appears to be CapTUI's primary weakness and will be discussed further in the next section. The majority of participants agreed that the angle visualizations were easy to understand. All participants agreed that the length visualization was easy to understand and that the length and angle visualizations were useful. Ten participants agreed that the visual guides helped them to draw more accurately.

When asked what the best thing about CapTUI was, eleven participants answered that it was the visual guides ("angle and length indicators extremely useful", "It is easy to use and accurate. Also the visual guides are helpful and make the drawings easy"). When asked what the hardest thing about CapTUI was, nine participants mentioned either tangible detection or difficulty with fine adjustments ("detecting the tools was sometimes not accurate", "adjusting the drawing tools was a bit hard").

Overall comments from participants were: "CapTUI is much easier to use and more accurate because of the visual guides", "very good for people who need accurate measurements", "made it easy to draw accurate lines and shapes. Would probably be very useful if integrated into a CAD package", "If the intention is to produce accurate geometric drawings I think CapTUI is superior - however still a little tricky to use in terms of accuracy and fine tuning", "With a little improvement to the localization of the tangibles it will become a very useful tool for precise drawings". 
In summary, CapTUI's main strength is in the ability to produce precise geometric constructions using the visual guides. Evidence of CapTUI's accuracy was shown by its significantly lower average angle error. Participants rated CapTUI significantly higher than Paint overall, enjoyed using CapTUI significantly more and found it to produce significantly more tidy drawings than Paint.

\section{Discussion}

There is very little work with tangibles for capacitive screens that demand a high level of interaction with precise tracking. In other projects the various blocks, dials and sliders are rotated and tracked, however the CapTUI drawing tools are manipulated in a more fine grained fashion, where a single degree of rotation is significant to the user. The most common feedback received from participants during our study was that they found the visual guides in CapTUI to be extremely useful for drawing precisely; however, making fine grained movements with the tangibles was difficult.

Designing tangibles for ease of use on a capacitive screen is a difficult problem. Of the user studies which feature tangibles on capacitive screens [26, 27], both acknowledge tangible design as a challenge; our tangibles are no different. Although our final tangibles were the best of all our designs, there are two remaining issues: consistent detection and accurate positioning.

For consistent detection there must be unbroken body-to-tangible contact and tangible-to-screen contact. A partial solution to this problem is to power the tangibles with their own battery, similar to the work in [9]. This would eliminate the need for the body-to-tangible contact. Maintaining the tangible-to screen contact would require a stable tangible design to ensure all touch points are on the screen at all times. There are also hardware and operating system limitations to consider such as enabling simultaneous touch and stylus input. Although this has been demonstrated in recent research [34], the ability to use such technology is limited to very few devices. Most devices, including the tablet used in our study, are not able to detect touch and stylus simultaneously. This means that if the stylus is on or in range of the screen (as a stylus can still be detected when it is a short distance away from the screen) then the tangibles cannot be detected via touch. Participants were informed of this limitation; however it still caused some problems when drawing.

A powered tangible is unlikely to solve the issue of accurate positioning. The main limitation here is the hardware. Capacitive hardware is designed to detect a general area of touch from a finger rather than a precise point. Our tangible touch points mimic this area of touch, however in order to calculate an accurate position and orientation of a tangible what is needed is a more precise point of touch. Currently the area of the touch point that is detected is translated to a centre point, but there is no way to know which part of the tangible's touch point is detected as the centre.

To assist in precise drawing it is possible to make more affordances in the software, similar to freezing the angle visualization (Section 3.4). However there is a fine line between tuning affordances to be helpful rather than causing frustration as the users control of the interface with the tangibles lessens. If they are not tuned carefully users may find the tangibles to be unresponsive and hinder them in achieving their 
goals. For example, we tried using automatic snaps to the horizontal and vertical screen positions of the tangible outline if the tangible came 'close' to these positions. Informal tests showed this to cause frustration when users did not want the tangibles in these positions. For example when tracking the tangible to a new position, if it was horizontal or vertical on the way to its new location, the tangible would seem unresponsive for some time due to the automatic snapping. We also experimented with averaging across three touch point positions to find a single position; however this did not always result in accurate positioning and caused delay in the recognition. Other possibilities would be allowing the user to lock the tangible outline in place or have a degree of stickiness to the movement; however such functions take away from overall goal of controlling the interface with the tangible.

If the above recognition issues can be resolved we believe a tangible drawing system would have great potential. Compared to more complicated drawing programs, tangibles are familiar and intuitive to use, don't require training, don't require the user to look at the tangible markings, therefore providing more flexibility. Our study showed that the main contributor to providing such an environment is the use of visual guides; without these the interaction is cumbersome. They provide essential feedback, such as the tangible outline, and additional information such as length and angle indicators. Users found our visual guides to be helpful and easy to understand; confirming that they enhance the interaction for geometric drawing. In future work we plan to compare tangible drawing systems to pen and paper.

\section{Conclusion}

We have presented CapTUI, a tangible drawing application for geometric constructions on a capacitive touch screen. CapTUI is composed of tangible drawing tools, a recogniser to identify each tool, a drawing application and visual drawing guides to augment the drawing experience. Our user evaluation compared CapTUI to Paint, which used non-recognizable drawing tools. Our results show that participants prefer CapTUI significantly more overall. Participants especially found the visual drawing guides to be helpful to drawing more precisely. The main challenges for tangibles on capacitive screens remain in good tangible design and consistent recognition.

Acknowledgements. This project is funded by the Royal Society of New Zealand Rutherford Foundation.

\section{References}

1. Marzola, E.S.: Using Manipulatives in Math Instruction. Journal of Reading, Writing, and Learning Disabilities International 3, 9-20 (1987)

2. Resnick, M., Martin, F., Berg, R., Borovoy, R., Colella, V., Kramer, K., Silverman, B.: Digital manipulatives: new toys to think with. In: Proceedings of the SIGCHI Conference on Human Factors in Computing Systems, pp. 281-287. ACM Press/Addison-Wesley Publishing Co. (1998) 
3. Uttal, D.H., Scudder, K.V., DeLoache, J.S.: Manipulatives as symbols: A new perspective on the use of concrete objects to teach mathematics. Journal of Applied Developmental Psychology 18, 37-54 (1997)

4. Tuddenham, P., Kirk, D., Izadi, S.: Graspables revisited: multi-touch vs. tangible input for tabletop displays in acquisition and manipulation tasks. In: Proceedings of the International Conference on Human Factors in Computing Systems, pp. 2223-2232. ACM, New York (2010)

5. Ullmer, B., Ishii, H.: The metaDESK: models and prototypes for tangible user interfaces. In: Proceedings of User Interface Software and Technology, pp. 223-232. ACM, New York (1997)

6. Baudisch, P., Becker, T., Rudeck, F.: Lumino: tangible blocks for tabletop computers based on glass fiber bundles. In: Proceedings of the International Conference on Human Factors in Computing Systems, pp. 1165-1174 (2010)

7. Wellner, P.: The DigitalDesk calculator: tangible manipulation on a desk top display. In: Proceedings of User Interface Software and Technology, pp. 27-33. ACM, New York (1991)

8. Fitzmaurice, G.W., Ishii, H., Buxton, W.: Bricks: Laying the Foundations for Graspable User Interfaces. In: Proceedings of CHI 1995, pp. 442-449. ACM Press/Addison-Wesley Publishing Co., New York (1995)

9. Yu, N.-H., Chan, L.-W., Lau, S.Y., Tsai, S.-S., Hsiao, I.-C., Tsai, D.-J., Hsiao, F.-I., Cheng, L.-P., Chen, M., Huang, P., Hung, Y.-P.: TUIC: enabling tangible interaction on capacitive multi-touch displays. In: Proceedings of the Conference on Human Factors in Computing Systems, pp. 2995-3004. ACM, New York (2011)

10. Inhelder, B., Piaget, J.: The early growth of logic in the child. Harper \& Row, New York (1964)

11. Piaget, J.: Play, dreams, and imitation in childhood. Norton, New York (1962)

12. Fails, J.A., Druin, A., Guha, M.L., Chipman, G., Simms, S., Churaman, W.: Child's play: a comparison of desktop and physical interactive environments. In: Proceedings of Interaction Design and Children, pp. 48-55. ACM, New York (2005)

13. Sitdhisanguan, K., Dechaboon, A., Chotikakamthorn, N., Out, P.: Comparative study of WIMP and tangible user interfaces in training shape matching skill for autistic children. In: TENCON 2007, pp. 1-4 (2007)

14. Chipman, G., Fails, J.A., Druin, A., Guha, M.L.: Paper vs. tablet computers: a comparative study using Tangible Flags. In: Proceedings of the International Conference on Interaction Design and Children, pp. 29-36. ACM, New York (2011)

15. Olson, I.C., Leong, Z.A., Wilensky, U., Horn, M.S.: It's just a toolbar!: using tangibles to help children manage conflict around a multi-touch tabletop. In: Proceedings of Tangible, Embedded, and Embodied Interaction, pp. 29-36. ACM, New York (2010)

16. Schneider, B., Jermann, P., Zufferey, G., Dillenbourg, P.: Benefits of a Tangible Interface for Collaborative Learning and Interaction. IEEE Trans. Learn. Technol. 4(3), 222-232 (2011)

17. Druin, A., Montemayor, J., Hendler, J., McAlister, B., Boltman, A., Fiterman, E., Plaisant, A., Kruskal, A., Olsen, H., Revett, I., Schwenn, T.P., Sumida, L., Wagner, R.: Designing PETS: A personal electronic teller of stories. In: Proceedings of the SIGCHI Conference on Human Factors in Computing Systems, pp. 326-329. ACM, New York (1999)

18. Karime, A., Hossain, M.A., Gueaieb, W., Saddik, A.E.: Magic stick: a tangible interface for the edutainment of young children. In: Proceedings of the IEEE International Conference on Multimedia and Expo, pp. 1338-1341. IEEE Press, Piscataway (2009) 
19. Xie, L., Antle, A.N., Motamedi, N.: Are tangibles more fun?: comparing children's enjoyment and engagement using physical, graphical and tangible user interfaces. In: Proceedings of Tangible and Embedded Interaction, pp. 191-198. ACM, New York (2008)

20. Price, S., Sheridan, J.G., Pontual Falcão, T., Roussos, G.: Towards a Framework for Investigating Tangible Environments for Learning. International Journal of Arts and Technology Special Issue on Tangible and Embedded Interaction 1(3/4), 351-368 (2008)

21. Scarlatos, L.L.: Tangible Math. Special Issue on Computer Game-Based Learning. International Journal of Interactive Technology and Smart Education 3(4), 293-309 (2006)

22. Hohenwarter, M., Jones, K.: Ways of linking geometry and algebra: the case of Geogebra. Proceedings of the British Society for Research into Learning Mathematics 27(3), 126-131 (2007)

23. Straesser, R.: Cabri-géomètre: Does Dynamic Geometry Software (DGS) Change Geometry and its Teaching and Learning? International Journal of Computers for Mathematical Learning 6, 319-333 (2002)

24. Rekimoto, J.: SmartSkin: an infrastructure for freehand manipulation on interactive surfaces. In: Proceedings of the SIGCHI Conference on Human Factors in Computing Systems, pp. 113-120. ACM (2002)

25. Chan, L., Müller, S., Roudaut, A., Baudisch, P.: CapStones and ZebraWidgets: sensing stacks of building blocks, dials and sliders on capacitive touch screens. In: Proceedings of the ACM Conference on Human Factors in Computing Systems, pp. 2189-2192. ACM, New York (2012)

26. Kratz, S., Westermann, T., Rohs, M., Essl, G.: CapWidgets: tangible widgets versus multitouch controls on mobile devices. In: Extended Abstracts on Human Factors in Computing Systems, pp. 1351-1356. ACM (2011)

27. Jansen, Y., Dragicevic, P., Fekete, J.-D.: Tangible remote controllers for wall-size displays. In: Proceedings of the ACM Conference on Human Factors in Computing Systems, pp. 2865-2874. ACM (2012)

28. Sutherland, I.E.: Sketchpad: A man-machine graphical communication system. In: Spring Joint Computer Conference: American Federation Information Processing Societies, pp. 329-346 (1963)

29. Balakrishnan, R., Fitzmaurice, G., Kurtenbach, G., Buxton, W.: Digital tape drawing. In: Proceedings of User Interface Software and Technology, pp. 161-169 (1999)

30. Couture, N., Riviere, G., Reuter, P.: GeoTUI: a tangible user interface for geoscience. In: Proceedings of Tangible and Embedded Interaction, pp. 89-96. ACM (2008)

31. Wiethoff, A., Schneider, H., Rohs, M., Butz, A., Greenberg, S.: Sketch-a-TUI: low cost prototyping of tangible interactions using cardboard and conductive ink. In: Proceedings of the International Conference on Tangible, Embedded and Embodied Interaction, pp. 309-312. ACM (2012)

32. Hse, H., Newton, A.R.: Recognition and Beautification of Multi-Stroke Symbols in Digital Ink. In: AAAI Fall Symposium Series, pp. 78-84 (2004)

33. Blagojevic, R., Chen, X., Tan, R., Sheehan, R., Plimmer, B.: Using tangible drawing tools on a capacitive multi-touch display. In: Proc. BCS-HCI 2012, pp. 315-320. British Computer Society (2012)

34. Hinckley, K., Yatani, K., Pahud, M., Coddington, N., Rodenhouse, J., Wilson, A., Benko, H., Buxton, B.: Pen + touch $=$ new tools. In: Proceedings of the ACM Symposium on User Interface Software and Technology, pp. 27-36 (2010) 